\section{El impacto de la Covid-19 en los destinos internacionales del Perú y opciones de política pública}

\author{
The impact of Covid-19 on international destinations of Peru \\ and public policy options
}

\begin{abstract}
RESUMEN
En un evento sin precedentes de una repercusión mundial e intersectorial, el sector servicios -por su implicancia inherente de interacción directa- ha sido uno de los más afectados, en especial la industria de viajes. Las proyecciones actuales avizoran una recuperación paulatina cargada de mucho desconcierto. Un factor fundamental que incide en el hábito de viajar proviene de los ingresos económicos. Esto, según proyecciones para América Latina y el Caribe, vería una pérdida considerable en progresos alcanzados en las últimas dos décadas. Así, el volumen de la demanda interna e interregional potencial vería una reducción sustantiva. En tal panorama, los destinos donde la cuota de mercado es preponderantemente internacional serían los más afligidos. De ahí la necesidad de políticas públicas sobre tales espacios geográficos y sus agentes económicos. Así, el presente artículo lista opciones de política pública en el ámbito laboral, financiero, demanda interna, así como oportunidades de mercado en un horizonte pospandemia.
\end{abstract}

Palabras Clave: Políticas públicas; agentes económicos; pospandemia; sector viajes.

\begin{abstract}
In an unprecedented event of global and intersectoral repercussion, the service sector - due to its inherent implication of direct interaction - has been one of the most affected, with the travel industry standing out. Current projections foresee a gradual recovery fraught with much confusion. A fundamental factor that affects the habit of traveling comes from economic income. This, according to projections for Latin America and the Caribbean, would see a considerable loss in the progress achieved in the last two decades. Thus, the volume of potential domestic and interregional demand would see a substantial reduction. In such a scenario, the destinations where the market share is predominantly international would be the most affected. Hence the need for public policies on such geographic spaces and their economic agents. Thus, this article lists public policy options in the
\end{abstract}

\section{Albert Moises Ponce Villegas}

albert.ponce@unmsm.edu.pe ORCID: https://orcid.org/00000002-1454-9200

Universidad Nacional Mayor de San Marcos. Lima, Perú

Presentado: 08/03/2021 - Aceptado: 06/08/2021 - Publicado: 23/12/2021

(C) Los autores. Este artículo es publicado por la revista Gestión en el Tercer Milenio de la Facultad de Ciencias Administrativas Universidad Nacional Mayor de San Marcos. Este es un artículo de acceso abierto, distribuido bajo los términos de la licencia Creative Commons Atribución 4.0 Internacional (CC BY 4.0) [https://creativecommons.org/licenses/by/4.0/deed.es] que permite el uso, distribución y reproducción en cualquier medio, siempre que la obra original sea debidamente citada de su fuente original. 
labour, financial, domestic demand, as well as market opportunities in a post-pandemic horizon.

Keywords: Public policies; economic agents; post-pandemic; travel sector.

\section{INTRODUCCIÓN}

Desde la década de los 50's, la evolución y crecimiento del sector viajes ha estado enmarcado por un desempeño constante y con pocos atisbos de estanco, llegando a representar el 7\% de las exportaciones mundiales (Organización Mundial del Turismo [OMT], 2017). En paralelo, estos guarismos positivos también se manifestaban en el plano nacional, tanto en el mediano como en el largo plazo (Centro Nacional de Planeamiento Estratégico [Ceplan], 2016) con valores cercanos al 4\% del PBI nacional (Ministerio de Comercio Exterior y Turismo [Mincetur], 2016).

Sin embargo, el 6 de marzo de 2020 será recordado como el inicio de un evento imborrable. Aquel día se conoció oficialmente que el SARS-CoV-2 ya se encontraba en nuestro territorio. En los meses siguientes, principalmente debido a nuestra poca capacidad hospitalaria -que a inicios de abril de 2020 solo se contaba con 99 unidades de cuidado intensivo (UCI) ${ }^{1}$ - el gobierno decidió establecer medidas estrictas de distanciamiento social. Esto conllevo a una parálisis de la economía nacional que, por ejemplo, en el mes de abril llegó a contraerse en un $39.9 \%{ }^{2}$. En efecto, sectores económicos como el comercio, servicios, minería o construcción se vieron paralizados. Si bien cada uno de ellos ha ido reactivándose en consonancia a una de las 4 fases de reactivación económica establecidas por el gobierno, estas medidas de apertura económica han tenido que paralizarse debido a disposiciones de una segunda cuarentena establecidas a inicios de este año.

Ahora bien, la reactivación del sector turismo es un tanto particular puesto que las perspectivas de recuperación básicamente descansan en un retorno cercano a la normalidad. Esto solamente se podrán alcanzar -ahora que se cuentan con vacunas disponibles y contratos

\footnotetext{
1 Para más información, consultar Sistema hospitalario (https:// opencovid-peru.com/reportes/sistema-hospitalario/)

2 Para más información, consultar Impacto del covid-19 en Perú y Latinoamérica (https://www.ipe.org.pe/portal/boletin-ipe-impacto-del-covid-19-la-economia-peruana-y-latinoamerica/)
}

efectivos- mediante la vacunación masiva de nuestros conciudadanos que permita alcanzar la denominada inmunidad de rebaño. Sin embargo, esto no parece muy cercano. The Economist (2021) proyecta -en relación a países de la región- que para mediados del año 2022 países como Perú, Brasil, Chile y Brasil estarían alcanzado la inoculación generalizada. Además, para fines de año se estarían incorporando Uruguay, Ecuador y Colombia. Finalmente, para mediados del 2023 se añadirían Bolivia, Paraguay y Venezuela. En suma, tanto a nivel nacional como regional no se vislumbra un escenario similar a la tan añorada normalidad: al menos en el corto plazo. Dentro de ello, hay que considerar actores y espacios geográficos con una mayor afectación puesto que su recuperación implicaría una recuperación más pausada. Probablemente el caso más resaltante sean los destinos nacionales de vocación y predominio del mercado internacional. Por ese lado, Perú cuenta con 9 atractivos -en su mayoría de jerarquía 3- con una presencia mínima de un $70 \%$ del mercado internacional.

En este artículo se desarrolla un análisis sobre los impactos más sustantivos que se desencadenarían en los destinos internacionales. En paralelo, se enumeran un conjunto de medidas de política pública por rubro económico que se podrían implementar, considerando criterios de impacto, relevancia y know-how.

\section{OBJETIVO DEL ARTÍCULO}

- Enumerar los impactos económicos de la pandemia en destinos internacionales.

- Catalogar un conjunto de medidas de política pública en destinos internacionales.

\section{ARGUMENTACIÓN}

El remesón económico provocado por la Covid-19 en muchas actividades económicas tendrá hasta ahora efectos indescifrables, pero previsiblemente disruptivos. En términos concretos, sobresale la reducción del 80\% del mercado internacional (Organización para 
la Cooperación y el Desarrollo Económicos [OCDE], 2020). Además, esta contracción en nuestro país -si no se cuentan a los visitantes en los meses iniciales previos a la pandemiase eleva al $99 \%$ con solo $50,951^{3}$ de visitantes internacionales.

Mirani (2021) proyecta que la recuperación económica del turismo pasa inicialmente por el mercado interno seguido del mercado regional. Respecto a lo último, un porcentaje alto del mercado internacional lo constituyen nuestros vecinos regionales como Chile, Ecuador, Colombia, Argentina y Brasil, representando 1 de cada 2 visitantes internacionales (Abedrapo et al., 2020). No obstante, en proyecciones del Banco Mundial, la recuperación económica de tales países -y el nuestro- no pareciera tomar una forma de «V» (Tabla 1). Dicho en otras palabras, el rebote económico no sería de un año para el otro.

Tabla 1

Proyecciones económicas en países de América Latina

\begin{tabular}{lccc}
\hline País & $\mathbf{2 0 1 9}$ & $\mathbf{2 0 2 0}$ & $\mathbf{2 0 2 1}$ \\
\hline Chile & $1.1 \%$ & $-4.3 \%$ & $3.1 \%$ \\
Ecuador & $0.1 \%$ & $-7.4 \%$ & $4.1 \%$ \\
Colombia & $3.3 \%$ & $-4.9 \%$ & $3.6 \%$ \\
Argentina & $-2.2 \%$ & $-7.3 \%$ & $2.1 \%$ \\
Brasil & $1.1 \%$ & $-8 \%$ & $2.2 \%$ \\
Perú & $2.2 \%$ & $-12 \%$ & $7 \%$ \\
\hline
\end{tabular}

Nota. Adaptación de Global Economic Prospects por World Bank [Banco Mundial], Junio 2020, World Bank Group, p. 86 (https:// doi.org/10.1596/978-1-4648-1553-9)

Esto es importante debido a que el tema económico es un componente fundamental que incide en la formación del hábito de viajar en las personas (Boto, 2020). En perspectiva, los dos mercados que podrían empujar la anhelada recuperación económica no parecen mostrar signos de recuperación, al menos en el horizonte próximo. Sin embargo, esto podría cambiar con la vacunación masiva, pero tal como se expuso al inicio de este artículo, el proceso tomaría tiempo y en el ínterin pueden implementarse medidas de confinamiento. Por ejemplo, al momento de redactar este artículo, el Perú estaba culminando un segundo confinamiento. Esto frenaría y deprimiría oferta y demanda.

3 Para mayor información, consultar Flujo de turistas internacionales e ingreso de divisas por turismo receptivo (https://datosturismo.mincetur.gob.pe/appdatosTurismo/Content1.html)
Adicionalmente, debemos tener en cuenta que este remezón económico va a provocar retrocesos sustantivos en el poder adquisitivo de las personas. Por ejemplo, el Programa de las Naciones Unidas para el Desarrollo bosqueja la primera caída del desarrollo humano desde que el término fue acuñado en la década de los 90's (Programa de las Naciones Unidas para el Desarrollo [PNUD], 2020). Esto significa que la pobreza en la región se incrementaría hasta un $34.7 \%$, valor cercano a dos veces la población de Chile o cuatro veces la de Bolivia. Esto implicaría un retroceso de hasta 10 años en el avance contra la pobreza en suelo peruano (Lavado y Liendo, 2020). Al respecto se puede vislumbrar que el poderío económico de potenciales visitantes tanto regionales como nacionales se verá mellado, inclusive podría volverse una actividad secundaria en los criterios presupuestarios de cada persona. Así, los destinos con predominio del mercado internacional necesitarían un mayor tiempo de recuperación y, por ende, los actores económicos de sus inmediaciones.

En nuestro país se da cuenta de 9 atractivos con presencia de más de un $65 \%$ del segmento internacional. Los mismos en su mayoría están jerarquizados de categoría 3 y son definidos como "recursos turísticos con rasgos excepcionales, capaces de motivar, por sí solos o en conjunto con otros recursos contiguos, un flujo actual o potencial de visitantes nacionales y/o extranjeros" (Mincetur, 2018, p.39). Tales atractivos se encuentran diseminados en cuatro regiones del país: Puno, Cusco, Madre de Dios y Loreto.

Como se observa en la Tabla 2, el promedio de visitantes extranjeros en Islas Uros, Choquequirao, Amantani y Pacaya Samiria representan entre el $68 \%$ y el $80 \%$. Esta cifra se eleva en Taquile, Tambopata y Raqchi a un rango de 80 y 85\%. Finalmente, la parte de Camino Inca sobrepasa el 98\%. Para comprender el impacto sobre tales jurisdicciones es preciso partir por el análisis en los agentes económicos (sectorialmente definidos como prestadores de servicios turísticos) y su dinámica empresarial en el mercado laboral.

\section{Empleo e informalidad}

En principio, de acuerdo con el Observatorio Laboral Covid-19 entre el período febrero 
Tabla 2

Atractivos nacionales con predominio del mercado internacional

\begin{tabular}{|c|c|c|c|c|c|c|c|c|c|}
\hline \multirow{2}{*}{ Atractivo } & \multicolumn{7}{|c|}{ Año (\% de visitantes internacionales) } & \multirow{2}{*}{ Promedio } & \multirow{2}{*}{ Jerarquía } \\
\hline & 2020 & 2019 & 2018 & 2017 & 2016 & 2015 & 2014 & & \\
\hline Islas Uros & 48 & 55 & 61 & 70 & 69 & 69 & 83 & $68 \%$ & 3 \\
\hline P. Arqueológico Choquequirao & 59 & 66 & 76 & 74 & 79 & 75 & 63 & $72 \%$ & 3 \\
\hline Islas Amantani & 64 & 76 & 78 & 80 & 74 & 78 & 83 & $78 \%$ & 2 \\
\hline Reserva Pacaya Samiria & 89 & 64 & 89 & 81 & 89 & 91 & 51 & $78 \%$ & 3 \\
\hline Islas Taquile & 74 & 80 & 82 & 83 & 79 & 82 & 83 & $82 \%$ & 2 \\
\hline R. Nacional de Tambopata & 65 & 83 & 83 & 84 & 85 & 87 & 88 & $85 \%$ & 3 \\
\hline Parque Arqueológico Raqchi & 58 & 79 & 81 & 83 & 86 & 94 & 86 & $85 \%$ & 3 \\
\hline $\begin{array}{l}\text { Camino Inka } \\
\text { (Sector Chachabamba, km. 104) }\end{array}$ & 0.2 & 99 & 99 & 99 & 96 & 99 & 100 & $99 \%$ & 3 \\
\hline $\begin{array}{l}\text { Camino Inka } \\
\text { (Qorywayrachina, km. 88) }\end{array}$ & 100 & 99 & 97 & 100 & 100 & 96 & 100 & $99 \%$ & 3 \\
\hline
\end{tabular}

Nota. El gráfico representa el porcentaje del mercado receptivo en comparación al total de visitantes. Sin embargo, el promedio está expresado tomando el período 2014-2019 por las posibles variaciones no uniformes del año 2020. Adaptado de Compendio de cifras de turismo por Mincetur, 2021, pp. 72, 73, 77, 79, 129, 130, 136, 137, 138 (https://bit.ly/3lrom6P).

2020/2021, en Latinoamérica se han perdido un total de 15.56 millones de empleos (Banco Interamericano de Desarrollo, 2021). En suelo peruano, la situación más intrincada fue la de junio de 2020 con una reducción de 2.8 millones de empleos. Si bien la situación ha ido mejorando con el pasar de los meses, aún persiste una brecha de medio millón de empleos en comparación a febrero de 2020, principalmente en el sector formal. Por ese lado, es probable que los prestadores de servicios se hayan visto en la necesidad de reinventar y desempeñarse en otras actividades económicas, aunque hay certeza estadística para señalar que estos rubros económicos alternativos se estarían dando en el lado informal.

Según se sugiere, la actual crisis estaría exacerbando el incremento de la informalidad a tasas más elevadas, pero tales impactos se estarían constatando con mayor firmeza en determinados estratos. Por un lado, el de las mujeres, debido a la mayor probabilidad que tienen de insertarse laboralmente en firmas informales y de salarios bajos (Sosale et al., 2021). Esto es relevante puesto que en el sector turismo las mujeres representan un 54\% del empleo total (World Tourism Organization [UNWTO], 2020). Otro ámbito de preocupación debe ser el rural donde podría estar incrementándose el trabajo familiar no remunerado (Ñopo y Pajita, 2020).
Sin embargo, de no haber migrado a operaciones informales, es probable que se estén posicionando en actividades fuera de la ley como la minería o tala ilegal. Cabe resaltar que tales ocupaciones cuentan con una mayor fuerza de atracción en la región amazónica. Finalmente, lo más peligroso es que este desplazamiento temporal termine convirtiéndose en una actividad permanente y estable. Sin el desarrollo de reformas por parte del nuevo gobierno, el sector informal e ilegal podría estar capturando mayor participación de la que cuenta. Esto afectaría indirectamente a los destinos en mención tanto en el mediano y largo plazo en tres aspectos: a) persistencia de brechas en prejuicio de segmentos menos favorecidos, b) incremento de la precariedad laboral y c) afectación del entorno natural y sus respectivos servicios ecosistémicos.

\section{¿Qué se podría hacer?}

Si bien alcanzar consensos sobre temas complejos como los mencionados requerirá un tira y afloja constante entre Ejecutivo y Legislativo, existen temas de común agenda sobre los cuales se puede comenzar con cierta celeridad como es el caso de la informalidad. Al respecto, Frisancho et al. (2020) -entre varios planteamientos- sugieren incrementar los costos de la formalidad, pero los mismos que estén anclados a incrementos de productividad y no al tamaño de la empresa. Además, los autores proponen 
el cambio del impuesto a la renta en personas jurídicas hacia uno similar en estructura al de personas naturales.

Además, otro aspecto por desarrollar debería ser la identificación de un sector informal con un alto potencial de mutar a la formalidad con mayor soltura. Para tal, se podrían implementar medidas de simplificación administrativa, fortalecimiento de capacidades, transferencia tecnológica, financiamiento o asociatividad empresarial en función de las características de cada unidad empresarial (Ghezzi, 2019), esto implicaría desenterrar ciertas visiones ortodoxas de que la formalidad es unidimensional o unimodal (Rigolini y Chacaltana, 2018). Finalmente, una medida mediata de empleo temporal se está dando por medio del programa Arranca Perú. La misma podría extender e incorporarse a sitios de interés turístico de una jerarquía mayor a 3.

\section{Apoyo gubernamental}

La mitigación de los estragos de la pandemia se relaciona con el nivel de significancia de la ayuda gubernamental. Por ese lado, el gobierno nacional implementó algunas medidas, entre las cuales destacan a) el subsidio a las planillas a salarios mensuales menores a $S / 1500$, b) el otorgamiento de capital de trabajo a través de fondos con garantía estatal como Reactiva Perú o FAE-Mype con el fin de evitar la ruptura en la cadena de pagos. Posteriormente salieron fondos de apoyo más sectoriales como el FAE-Agro o el FAE-Turismo. Si bien no se conoce exactamente la proporción sectorial con la cual se repartió los fondos, el apoyo ha llegado a una gran número de micro y pequeñas empresas. Por un lado, hasta setiembre del 2020 más del $60 \%$ del fondo Reactiva llegó a cubrir a empresas con menos de 50 trabajadores lo que equivale a un millón 229 mil de puestos de trabajo (Banco Central de Reserva, 2020). Por el otro, a junio de 2020 el programa FAE-Mype llegó a cubrir a más 140 mil empresas beneficiarias ${ }^{4}$. En paralelo, a inicios de esta pandemia el gobierno mediante el Decreto de Urgencia 076-2020 inyectó capital al fondo concursable Turismo Emprende por un valor de 50 millones de soles, el mismo que excede en 5 veces el monto total del

\footnotetext{
4 Para mayor información, consultar Estadísticas del FAE-Mype (https://cdn.www.gob.pe/uploads/document/file/862441/Estadi\%CC\%81sticas_FAE_MYPE.pdf).
}

fondo. Este financiamiento busca apoyar a las pymes en su reconversión económica a través de iniciativas de innovación, adecuación sanitaria y tecnológica.

\section{¿Qué se podría hacer?}

En primer lugar, si bien la cobertura de ambos fondos con garantía estatal oscila entre el 80 al 98\%, Aparicio y Balarez (2020) proponían que tal garantía se extendiera al $100 \%$ en el sector turismo y que no existiera diferencia alguna entre los distintos volúmenes de crédito. Adicional a ello, el gobierno en recientes semanas ha anunciado una nueva convocatoria del fondo Turismo Emprende. El mismo tiene algunas adiciones encomiables sobre las que puede destinarse el fondo como el pago a proveedores, adquisición de equipamiento, capacitación o promoción. Por ejemplo, un artículo muestra que las empresas que invirtieron mayores volúmenes en promoción en la recesión de 1919 en los EE.UU. incrementaron sus ventas a una tasa mayor al de sus competidores (Ritson, 2020).

\section{Otros rubros económicos}

Asimismo, con el fin de dinamizar el sector de Alimentos y Bebidas (A\&B) se podría desarrollar un programa de vales de consumo que brinden descuentos, esto se realizó en el Reino Unido y ha tenido resultados superiores al de los esperados (The Economist, 2020a). Así, para el caso de alojamientos y plataformas colaborativas se puede tomar el ejemplo desarrollado en Lisboa (Portugal). El programa dota de mejores oportunidades de acceso a una vivienda en jóvenes y familias de bajos ingresos en las viviendas que ya atraen visitantes (O'Sullivan, 2020).

En nuestro país, esto podría canalizarse inicialmente a través de un mapeo e identificación de los propietarios que ofertan sus alojamientos en plataformas colaborativas. Posteriormente, se podría incluir, por ejemplo, al fondo MiVivienda y este asuma el rol de adquirir una parte -o toda- del valor del inmueble. En paralelo, para fomentar la demanda interna se podría implementar un bono de vacaciones destinado a familias de menores ingresos. Cabe resaltar que esto ya se viene implementando en Italia, Corea del Sur, Taiwan y Tailandia (The Economist, 2020b). Aunque previamente debería evaluarse el tema económico y el efecto 
multiplicador que desencadenaría sobre la economía. Finalmente, otro incentivo podría ser la reducción de la jornada laboral en determinadas fechas que coincidan con festividades o eventos en el territorio nacional relevantes, tal como se ha sugerido en Nueva Zelanda (El País, 2020).

Si bien hasta el momento no se avizora una recuperación rápida y próxima, esta crisis puede ser tomada como una oportunidad para repensar el turismo (OECD, 2020) y abordar aspectos que ya se venían sugiriendo con relativa urgencia como cambios en la parte regulatoria, legal y gobernanza (OECD, 2018). En virtud de ello, Mirani (2021) proyecta dos cambios fundamentales en relación a la industria de viajes. Primero, los viajes se darían con menor frecuencia, pero con un ligero incremento en los días de estadía, aspecto que diverge de tendencias prepandemia (Borden, 2019). Segundo, la naturaleza de los viajes podría redefinirse y se le podrían incorporar factores -hasta ahora insospechados- como el emparejamiento con el trabajo remoto o el impulso de acciones gubernamentales que busquen limitar el flujo de visitantes (Jamrisko et al., 2020). Sin embargo, un aspecto a no perder de vista es que estos cambios podrían configurar una nueva demanda más alineados al encarecimiento de los territorios. A manera ilustrativa, veamos el caso de Machu Picchu que, en un período prepandemia recibía un total de 5,600 personas distribuidas en dos turnos. Al respecto, Vilela et al. (2018) exponen que la cuota de mercado se distribuye en $70 \%$ para el internacional y $30 \%$ para el nacional. Esto implica que -en promedio- 3,920 turistas eran internacionales y 1,680 nacionales. Asimismo, los autores encuentran que el gasto diario promedio es de S/ 658 para el internacional y S/ 299 para el nacional. En términos de impacto económico, esto implicaría S/ $2,579,360$ por el lado internacional y S/ 502,320 de su contraparte nacional.

No obstante, en la próxima reapertura del santuario el 1 de marzo solo estará permitido un aforo del $40 \%{ }^{5}$. Esto implicaría el ingreso de solamente 2,240 personas $(1,568$ internacionales y 672 nacionales). Asumiendo un mismo

5 Para mayor información, consultar Machu Picchu reabre sus puertas a la visita de turistas a partir del 1 de marzo (https://cnnespanol.cnn.com/2021/02/28/machu-picchu-reabre-sus-puertas-a-la-visita-de-turistas-a-partir-del-1-de-marzo/). patrón de gasto, el impacto económico desencadenado por el turista internacional sería de $\mathrm{S} /$ $1,031,744$ y S/ 200,928 por parte del nacional. Esto arroja una pérdida estimada diaria de $\mathrm{S} /$ $1,547,616$ proveniente del mercado internacional y S/ 301,392 del nacional, lo que en términos porcentuales se expresa en una pérdida neta del $60 \%$ por conceptos de ingresos.

Vilela et al. (2018) mostraba que el número de empleos creados en Machu Picchu el año 2017 fue de 29,522. Con la reducción del 60\% en impacto económico -y asumiendo otras variables constantes- la demanda solamente podría cubrir y crear 11,809 empleos. Esta situación podría acarrear la quiebra de muchas empresas, ya que las mismas reducen sus costos para ofrecer precios módicos y competitivos para así captar y mantener su porción de mercado. Sin embargo, debe considerarse que es poco probable que otras variables se mantengan constantes, sino todo lo contrario: que cambien radicalmente como los asociados a estadía, restricciones de aforo, preferencias, capacidad o hábito de gasto.

\section{Reinvención de negocios}

En efecto, esta nueva normalidad debe invitar al diseño de nuevas medidas que permita generar nuevos nichos de mercado para los prestadores de servicios que podrían quedar fuera del mercado convencional. Así, se podría desarrollar políticas, teniendo de referencia acciones desarrollados alrededor del mundo. Así, por ejemplo, en la isla de Madeira (Portugal) se ha decidido por probar un piloto de aldea digital. La misma busca captar trabajadores tecnológicos a través de una oferta global entre alojamiento, entretenimiento y servicios (Tzvetozar, 2021). En seguida, un santuario de animales ha encontrado un camino de generar ingresos a través de sus principales activos: los animales. El santuario aprovechando el incremento de reuniones virtuales, ofrece incluir a una llama o una cabra dentro de una videollamada (Leskin, 2020) lo que ha provocado reservas aseguradas en más de 300 personas. Asimismo, el desarrollo de productos debe partir por espacios de colaboración que permitan arrojar y diseminar nuevas ideas que permitan generar productos con un alta cuota de innovación, como por ejemplo la convocatoria de escritores de ciencia ficción (Fleming, 
2019) y así ampliar el espectro de aristas que podríamos estar omitiendo en el diseño de experiencias. Así pues, se estaría contribuyendo a avizorar mercados y modelar productos bajo una mirada interdisciplinaria y de menor estacionalidad.

\section{Repensar el mercado}

Además, este período de cuarentena viene suprimiendo y limitando muchas de las actividades que realizamos que, a día de hoy, valoramos $\mathrm{y}$ le hemos reasignado un nuevo valor. A medida que vayamos superando paulatinamente esta crisis y, si todavía nuestra capacidad económica no ha sido afectada en demasía, es probable que crezca nuestros deseos de viajar y disfrutar de nuevos experiencias. Previamente, más de $50 \%$ millenials en 42 países del mundo mostraban su deseo de viajar por el mundo (Deloitte, 2019), así como un incremento en su insatisfacción laboral.

Ambos elementos pueden catapultar una demanda de viajes que se alineen a un propósito de restitución de la satisfacción pérdida producto de la actual crisis. Además, estas necesidades estarían alineadas a espacios alejados de las urbes como las áreas rurales o las áreas protegidas. Estos territorios tendrían dos ventajas sustantivas para ser posicionadas como altamente singulares. Por un lado, existe evidencia que muestra que pasar al menos 2 horas a la semana en espacios naturales está correlacionado a una buena salud (White et al., 2019). Asimismo, al ser espacios de gran extensión y, por ende, silentes, podría incrementar la productividad del trabajador (Dean, 2020).

\section{CONCLUSIONES}

Si bien en la actualidad nos encontramos en una situación sanitaria relativamente mejor que hace un año. Esta capacidad de análisis retrospectivo es muy difícil de aplicar al plano prospectivo. Más aún en estos momentos. Se sugiere que eventos de gran impacto como la actual pandemia van a modelar cambios perdurables. La dificultad radica en identificar sobre qué aspectos sociales, económicos, tecnológicos o políticos se va a dar. Lo relativamente certero es que hay estratos sociales más afectados. En el sector turismo, estos son los destinos internacionales y sus agentes económicos circunscritos. Si bien las propuestas referenciadas pueden extenderse a otros rubros económicos, esta debe ser una cruzada para proteger a los más vulnerables. Esto implica una participación gubernamental activa y una convocatoria multigeneracional que permita concretar políticas públicas enmarcadas en una visión de futuro donde prime y se fomente la sostenibilidad, la productividad, formalidad y la innovación. Para ello, se requiere políticas públicas de gran sapiencia, con vocación experimental, sustentada en datos y perfectible sobre la marcha. Parafraseando a Schumpeter, se nos está brindando una oportunidad para enterrar lo viejo e instaurar lo nuevo.

\section{REFERENCIAS BIBLIOGRÁFICAS}

Abedrapo, J., Arancibia, R., Del Prado, J., González, S., Eyzaguirre, R., Gamio, P., Macera, D., Montoya, J., Morandé, J., Ovando, C., Parodi, D., Riquelme, D., Stefoni, C., Valencia, R., Vásquez, T. (2020). Una visión de futuro de las relaciones chileno-peruanas. En S. Namihas (Ed.). Recuperado de http://www.generaciondedialogo.org/ images/2020/Una visión de futuro de las relaciones chileno-peruanas.pdf

Aparicio, C., y Balarez, N. (2020). Reactivando al sector turismo. Recuperado de Gestión: https:// gestion.pe/economia/reactivando-al-sector-turismo-apoyo-consultoria-economia-peruana-fea-mype-reactiva-peru-fae-turismo-noticia/

Banco Central de Reserva. (2020). Reactiva Perú. Recuperado de https://www.bcrp.gob.pe/ docs/Publicaciones/Reporte-Inflacion/2020/ setiembre/ri-setiembre-2020-recuadro-5.pdf

Banco Interamericano de Desarrollo. (2021). Observatorio Laboral Covid-19. Recuperado de https://observatoriolaboral.iadb.org/es/empleo/

Borden, T. (2019). Millennials are taking multiple "micro-cations" instead of long trips, and it says a lot about their work-life balance priorities. Recuperado de Business Insider: https://www. businessinsider.com/millennials-are-shortening-travel-and-leading-microcation-mini-vacation-trend-2019-12

Boto, D. (2020). Habit Formation in Tourism Traveling. Journal of Travel Research, 1-30. https:// doi.org/10.1177/0047287520964597

Centro Nacional de Planeamiento Estratégico. (2016). El Futuro del Turismo (p. 27). Recuperado de https://www.ceplan.gob.pe/wp-con- 
tent/uploads/2016/07/el-futuro-del-turismo-04.07.2016.pdf

Dean, J. (2020). Noise, Cognitive Function, and Worker Productivity. 1-84. https://joshuatdean. com/wp-content/uploads/2020/02/NoiseCognitiveFunctionandWorkerProductivity.pdf

Deloitte. (2019). The Deloitte Global Millennial Survey 2019: Societal discord and technological transformation create a "generation disrupted." En Deloitte. https://www2.deloitte.com/content/dam/ Deloitte/global/Documents/About-Deloitte/deloitte-2019-millennial-survey.pdf

El País. (2020). La primera ministra de Nueva Zelanda sugiere una semana laboral de cuatro días para reactivar la economía. Recuperado de: https://elpais.com/sociedad/2020-05-20/ la-primera-ministra-de-nueva-zelanda-sugiere-una-semana-laboral-de-cuatro-dias-para-reactivar-la-economia.html?ssm=TW_CM

Fleming, S. (2019). The French army is enlisting sci-fi writers to predict the future. Recuperado de World Economic Forum: https://www. weforum.org/agenda/2019/07/france-army-science-fiction-writers-global-risks

Frisancho, V., Lavado, P., Liendo, C., y Saavedra, J. C. (2020). Formalización del empleo, protección de familias y reducción de desigualdades. Recuperado de: https:// focoeconomico.org/2020/09/18/formalizacion-del-empleo-proteccion-de-familias-y-reduccion-de-desigualdades/

Ghezzi, P. (2019). Informalidad: una visión alternativa. Recuperado de Foco Económico: http:// focoeconomico.org/2019/02/08/informalidad-una-vision-alternativa/

Jamrisko, M., Thanthong, R., \& Alegado, S. (2020). Covid Gives Tourism Chance to Curb Future Environmental Damage. Recuperado de Bloomberg Green: https://www.bloomberg.com/ news/features/2020-10-14/covid-lets-thailand-philippines-new-zealand-curb-tourisms-eco-damage?utm_source=twitter\&utm_content=business\&cmpid=socialflow-twitter-business\&utm_medium=social\&utm_campaign=socialflow-organic

Lavado, P., y Liendo, C. (2020). COVID-19, pobreza monetaria y desigualdad. Recuperado de Foco Económico: http://focoeconomico. org/2020/05/29/covid-19-pobreza-monetaria-y-desigualdad/

Leskin, P. (2020). Invite a llama or goat to your next corporate Zoom meeting or video call for under \$100. Recuperado de Business Insider: https:// www.businessinsider.com/zoom-video-goat2-meeting-llama-sweet-farm-animals-cameo-2020-4

Ministerio de Comercio Exterior y Turismo. (2016). Medición Económica del Turismo. Recuperado de https://www.mincetur.gob.pe/wp-content/uploads/documentos/turismo/publicaciones/MEDICION_ECONOMICA_TURISMO_ ALTA.pdf

Ministerio de Comercio Exterior y Turismo. (2018). Manual para la elaboración y actualización del inventario de recursos turísticos. Recuperado de https://cdn.www.gob.pe/uploads/document/ file/401761/Manual_para_la_Elaboaracion_y_ actualizacion_del_inventario_de_recursos_turisticos.pdf

Ministerio de Comercio Exterior y Turismo. (2021). Perú: Compendio de cifras de turismo, junio 2021. Recuperado de https://cdn.www.gob. pe/uploads/document/file/2040432/Compendio $\% 20$ Cifras $\% 20$ de $\% 20$ Turismos $\% 20$ Junio\%202021.pdf

Mirani, L. (2021). Holidays in 2021 will be fewer, longer and closer to home. Recuperado de The Economist: https://www.economist.com/theworld-ahead/2020/11/16/holidays-in-2021will-be-fewer-longer-and-closer-to-home

Ñopo, H., y Pajita, D. (2020). Pandemia y Empleo Rural. Recuperado de Foco Económico: http://focoeconomico.org/2020/08/28/pandemia-y-empleo-rural/

O’Sullivan, F. (2020). Lisbon Has a Plan to Reclaim Housing From Airbnb. Recuperado de https://www.bloomberg.com/news/ articles/2020-07-08/lisbon-s-plan-to-reclaim-vacation-rentals-for-housing?utm medium =email\&_hsmi=91413380\&_hsenc $=$ p2ANqtz-8Y65JVEQkMTIeJwD7fPvHnRuN4hX8hjMk3Mher7MY43uuRzDqKJxnLW9dc_0rNC6_iMNiC4eKb6QhbM0kJERiVXtvmSVGbGkqzhHKK1

Organisation for Economic Co-operation and Development. (2018). Analysing Megatrends to Better Shape the Future of Tourism. OCDE Tourism Papers, (No. 2018/02), 0-69. https://doi. org/10.1787/d465eb68-en

Organisation for Economic Co-operation and Development. (2020). Rebuilding tourism for the future: COVID-19 policy responses and recovery. Recuperado de https://read.oecd-ilibrary.org/ view/?ref=137_137392-qsvjt75vnh\&title=Rebuilding-tourism-for-the-future-COVID-19-policy-response-and-recovery 
Organización Mundial del Turismo. (2017). Edición 2017 Panorama OMT del turismo internacional. https://doi.org/10.18111/9789284419043

Programa de las Naciones Unidas para el Desarrollo. (2020). COVID-19: el desarrollo humano va camino de retroceder este año por primera vez desde 1990. Recuperado de: https://www. undp.org/content/undp/es/home/news-centre/news/2020/COVID19_Human_development_on_course_to_decline_for_the_first_ time_since_1990.html

Rigolini, J., y Chacaltana, J. (2018). Debunking three myths about Informality. Recuperado de Banco Mundial Blogs: http://blogs.worldbank.org/ jobs/debunking-three-myths-about-informality

Ritson, M. (2020). The best marketers will be upping, not cutting, their budgets. Recuperado de Marketing Week: https://www.marketingweek.com/mark-ritson-marketing-spend-recession-coronavirus/

Sosale, S., Bunker, S., McDonald, L., \& Rubiano, E. (2021). Reconstruir mejor después de la COVID-19: promoviendo la igualdad de género en la educación y el desarrollo de habilidades para una mayor fuerza laboral. Recuperado de Banco Mundial Blogs: https://blogs.worldbank.org/ es/datos/reconstruir-mejor-despues-de-la-covid-19-promoviendo-la-igualdad-de-genero-en-la-educacion?cid=ECR_TT_worldbank_ ES_EXT

The Economist. (2020a). As "Eat out to help out" ends, Britain's eateries remain at risk. Recuperado de: https://www.economist.com/ graphic-detail/2020/08/31/as-eat-out-tohelp-out-ends-britains-eateries-remain-at-risk?utm_campaign=the-economist-today\&utm_ medium=newsletter\&utm_source=salesforce-marketing-cloud\&utm_ter m=2020-0901\&utm_content=article-link-4\&et

The Economist. (2020b). Why governments are paying people to go on holiday. Recuperado de: https://www.economist.com/international/2020/07/27/ why-governments-are-paying-people-to-go- on-holiday?utm_campaign=the-economist-today\&utm_medium=newsletter\&utm_source=salesforce-marketing-cloud\&utm_term=2020 07-28\&utm_content=article-link-6

The Economist. (2021). Vaccine nationalism means that poor countries will be left behind. Recuperado de https://www.economist.com/graphic-detail/2021/01/28/vaccine-nationalismmeans-that-poor-countries-will-be-left-behind

Tzvetozar, I. (2021). First 'digital village' springs up on the island of Madeira. Recuperado de The Mayor: https://www.themayor.eu/en/a/view/ first-digital-village-springs-up-on-the-islandof-madeira-7089

Vilela, T., Rubio, J. C., Escobedo, A. J., Bruner, A., y Conner, N. (2018). El Impacto Económico Local del Turismo En Áreas Naturales Protegidas del Perú. Recuperado de https://www.conservation-strategy.org/sites/default/files/field-file/ ES_Documento_de_trabajo_Peru_Tourism_ Multipliers_Feb_2018_0.pdf

White, M. P., Alcock, I., Grellier, J., Wheeler, B. W., Hartig, T., Warber, S. L., ... Fleming, L. E. (2019). Spending at least 120 minutes a week in nature is associated with good health and wellbeing. Scientific Reports, 9(1), 1-11. https://doi. org/10.1038/s41598-019-44097-3

World Tourism Organization. (2019). Global Report on Women in Tourism Second edition. https:// doi.org/10.18111/9789284420384

World Bank. (2020). Global Economic Prospects, June 2020. Washington, DC: World Bank. https://openknowledge.worldbank.org/handle/10986/33748 License: CC BY 3.0 IGO. 